\title{
Profile of cause of death assigned to adults on antiretroviral therapy in Soweto
}

\author{
A S Karstaedt
}

This retrospective cohort study describes causes of death in 305 patients (baseline median CD4 count 26/ $\mu$ l) from 2943 adults on antiretroviral therapy. Acute sepsis (20\%), tuberculosis (18\%) and Mycobacterium avium complex (MAC) bacteraemia (14\%) were the most common causes. Mortality owing to the disease was $66 \%$ for MAC bacteraemia and 23\% for non-Hodgkin's lymphoma. In 37 patients dying beyond one year on ART, virological failure was present in 11 (30\%), and non-HIV-related causes of death occurred in 10. The main causes were acute sepsis (6), tuberculosis (7) and chronic medical conditions (5). Initiating ART at higher CD4 counts should reduce early mortality.

S Afr Med J 2012;102(8):680-682. DOI:10.7196/SAMJ.5369
Early mortality on antiretroviral therapy (ART) in low-income countries is substantially higher than in high-income countries. ${ }^{1}$ Mortality during the first year on ART in sub-Saharan Africa has ranged from $8 \%$ to $26 \%{ }^{2}$ Main causes of early death in cohort studies from sub-Saharan Africa include tuberculosis, acute sepsis, cryptococcal meningitis, malignancies and chronic diarrhoea or wasting syndromes. ${ }^{2}$ Less is known about causes of death later in the course of ART. In high-income countries, there is increasing emphasis on non-HIV infection-related causes of death. ${ }^{3}$ This study describes the causes of death in a large single-centre cohort on ART according to time on treatment and, in particular, death beyond the first year on ART.

\section{Methods}

Chris Hani Baragwanath Hospital is a 2700 -bed university-associated public sector hospital serving Soweto. WHO stage 4 diseases (AIDS) or a CD4 cell count $\leq 200$ cells/ $\mu$ l qualify patients for ART, usually comprising stavudine, lamivudine and efavirenz. Cotrimoxazole prophylaxis was provided too. Frequent clinic visits were scheduled for the first 6 months, after which clinically well patients were seen less frequently.

A retrospective cohort study was conducted of the 2943 adult patients ( $\geq 18$ years of age) who initiated ART between 1 April 2004 and 31 December 2005. Patients were followed till January 2009 for a median of 2.7 years.

Causes of death were assigned by attending doctors in the hospital wards and HIV clinic. For patients who died at home, an attempt was made to differentiate sudden unexpected death from chronic ill-health. Family members of patients who died at home were informally asked the symptoms and cause of death as part of routine follow-up of patients who missed appointments or if family members reported the death. The researcher reviewed clinic records, inpatient hospital files and laboratory investigations on all patients who died, and designated a final cause of death. Acute sepsis included acute pneumonia, acute bacterial meningitis, septicaemia and other severe bacterial infections. Chronic medical conditions

Division of Infectious Diseases, Department of Medicine, Chris Hani Baragwanath Hospital and University of the Witwatersrand, Johannesburg A S Karstaedt, MB BCh, M Med included diabetes mellitus, chronic cardio-respiratory diseases and renal diseases (excluding HIV-associated nephropathy). Unmasking of an appropriate opportunistic disease within 6 months of starting effective ART was adjudged to be immune reconstitution disease (IRD). Patients were considered lost to follow-up if not seen in the 6 months preceding the close of the study.

The study was approved by the Committee for Research on Human Subjects of the University of the Witwatersrand.

Chi-squared test calculations or Fisher's exact test were used for group comparisons.

\section{Results}

Death was known to have occurred in 305 (10.4\%) of the 2943 patients. The median age of those who died was 36 years (range 19 - 69 years) and the median CD4 count at baseline was 26/ $\mu$ (range 1 479/ $\mu$ l). Table 1 compares baseline characteristics of 305 patients who died with the 2638 survivors. Comparing the baseline characteristics of the 268 patients who died $\leq 12$ months on ART with the 37 who died $>12$ months on ART, significantly more died in the first 12 months with CD4 <50 cells/ $\mu$ l $(193(72 \%)$ v. $18(49 \%)(p=0.0039))$ and with WHO stage 4 disease (168 (63\%) v. $12(32 \%)(p=0.0004))$.

Of those who died, 165 (54\%) died in the first 3 months on ART, $53(17 \%)$ in months $4-6,50(16 \%)$ in months $7-12,22(7 \%)$ in the second year, $9(3 \%)$ in the third year, and $6(2 \%)$ beyond 3 years. Over the study period, 268 (9.1\%) patients were lost to follow-up.

A specific cause of death was retrospectively assigned to 275 (90\%) patients. There was no information on $22(7 \%)$ of the deaths, and 8 (3\%) had a sudden unexpected death at home. More than one cause was found in 14 patients.

Table 1. Baseline characteristics of patients who survived and those who died

\begin{tabular}{|c|c|c|c|}
\hline & $\begin{array}{l}\text { Surviving cohort } \\
N(\%)\end{array}$ & $\begin{array}{l}\text { Deaths } \\
N(\%)\end{array}$ & $p$-value \\
\hline Total number & 2638 & 305 & \\
\hline Male & 1030 (39\%) & $118(39 \%)$ & NS \\
\hline $\mathrm{CD} 4<50 / \mu \mathrm{l}$ & 936 (35\%) & $211(69 \%)$ & $<0.0001$ \\
\hline HIV-1 RNA & & & \\
\hline$\geq 100000$ copies $/ \mathrm{ml}$ & $1556(59 \%)$ & $221(72 \%)$ & $<0.0001$ \\
\hline WHO stage 3 & $1123(43 \%)$ & $84(28 \%)$ & $<0.0001$ \\
\hline WHO stage 4 & $615(23 \%)$ & $180(60 \%)$ & $<0.0001$ \\
\hline
\end{tabular}


Acute sepsis (20\%), TB (18\%) and Mycobacterium avium complex (MAC) bacteraemia (14\%) were the most common causes of death (Fig. 1). Of 64 with confirmed MAC bacteraemia in the total cohort, $42(66 \%)$ died from the condition. Standard initial treatment of MAC bacteraemia included clarithromycin with ethambutol and a third drug, usually ciprofloxacin. Lactic acidosis as a toxicity owing predominantly to stavudine was implicated in 11 (3.6\%) deaths. The 21 AIDS-defining cancers causing death comprised Kaposi's sarcoma (12), non-Hodgkin's lymphoma (6), Burkitt's lymphoma (1), and carcinoma of the cervix (2). Of 30 in the cohort with non-Hodgkin's lymphoma, 7 (23\%) died. The 7 non-AIDS defining malignancies were 3 with Hodgkin's lymphoma, and 1 each with carcinoma of the lung, anus and oesophagus, and one with acute myeloid leukaemia. Chronic medical conditions included 9 patients with cardiac disease (4 with dilated cardiomyopathy, 2 with rheumatic heart disease, and 1 each with atrial myxoma, third-degree heart block, and aortic aneurysm), 5 with chronic lung disease, 3 with chronic renal failure predating ART, and 3 with diabetes mellitus. Sepsis was microbiologically defined in 15 (25\%) patients with acute sepsis including 3 patients with bacterial meningitis (2 with Streptococcus pneumoniae and 1 with non-typhoidal salmonella who was also bacteraemic) and 13 with bacteraemia (5 with Escherichia coli, 4 with Staphylococcus aureus and 4 with non-typhoidal salmonella). Unmasking IRD comprised 4 patients with tuberculosis and 2 with MAC infection.

Deaths in hospital occurred in $39(78 \%)$ of 50 patients who died after 7 - 12 months on ART and in 26 (70\%) of 37 who died after $>12$ months on ART. Virological failure (viral load $>400$ copies/ml) was noted in $9(18 \%)$ who died in months $7-12$, and $11(30 \%)$ who died beyond the first year; 8 of the latter 11 died of sepsis, tuberculosis or MAC bacteraemia. Common causes of death $>12$ months after starting ART were acute sepsis (16\%), TB (19\%; 5 cultured; 1 each with multi-drug resistance and rifampicin monoresistance), and chronic medical conditions (14\%). NonHIV-related causes were found in 10 patients, comprising chronic medical conditions (5), motor vehicle accident (2), and 1 each with cancer of the anus, pulmonary embolism (in a patient with chronic diarrhoea related to intestinal spirochaetosis), and postoperative complications. Three patients died of lactic acidosis in the second year.

\section{Discussion}

The most striking finding of this study among AIDS-related causes of death, in contrast with other African studies, ${ }^{2,4}$ was the importance of MAC bacteraemia. It has been recognised as an important opportunistic infection in countries in sub-Saharan Africa in studies employing mycobacterial blood cultures. ${ }^{5-6}$ It is associated with a high mortality in the ART era with only $30 \%$ of such patients alive beyond 2 years. ${ }^{7}$ Treatment failure is common, reasons including drug toxicity, inadequate serum levels and development of drug resistance. Primary prophylaxis with clarithromycin or azithromycin for patients with CD4 cell counts $<50-100 / \mu$ l might have prevented cases prior to ART.

$\mathrm{TB}$ and cryptococcal meningitis have been the leading causes of death in most other African studies. ${ }^{2,4}$ Cryptococcal meningitis was a less common cause in this study., Bacterial causes of death, other than mycobacterial infections, have not been well-defined in ART studies. The organisms found are common in patients with HIV infection. ${ }^{2}$

Among non-Hodgkin's lymphoma patients co-infected with HIV in California, 59\% died within 2 years of diagnosis compared with $30 \%$ without HIV infection. ${ }^{8}$ In Uganda, only 20 of 154 patients with non-Hodgkin lymphoma, irrespective of HIV status, were known to be alive a year after diagnosis. ${ }^{9}$ The outcome in this study appears better but merits a dedicated study as some patients who died might not have accessed ART. Non-AIDS-defining cancers are likely to increase with longer duration on ART and increasing age.

Unmasking IRD was an uncommon cause of death, as it was in Uganda. ${ }^{4}$ Two missing causes of death, compared with other settings, ${ }^{3}$ were liver failure, possibly owing to low rates of hepatitis $\mathrm{C}$ infection in this cohort and the activity of lamivudine against hepatitis B, and ischaemic heart disease, since coronary atherosclerosis is uncommon in this population. ${ }^{10}$

This study mirrored the high first-year mortality in cohorts in sub-Saharan Africa. ${ }^{2,4}$ Mortality was low beyond 1 year on ART, as in similar studies. ${ }^{2,4}$ Beyond 12 months of ART, virological failure was a prominent finding in those who died. This was partially responsible for the continued importance of TB, MAC bacteraemia and acute sepsis, accounting for half of these cases. Non-HIV-related causes, especially chronic medical conditions, acquired increased relative prominence.

This study has some potential limitations. Many patients were lost to followup, some of whom probably died. ${ }^{11}$ Missed deaths might have affected the ranking of causes of death. No autopsies were performed. There was no formal verbal autopsy for those who died at home, and not all of those had a family member interviewed.

The study strength, despite its retrospective nature, was that ascertainment of cause of death was as accurate as could be achieved without autopsies. Those who died having received ART for $<6$ months were seen frequently as in- or outpatients.

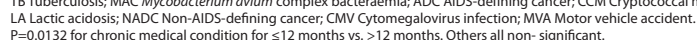

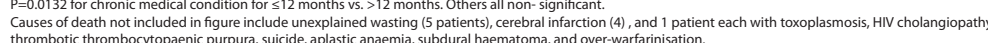

Fig. 1. Causes of death of 268 patients on $A R T \leq 12$ months and 37 patients on $A R T>12$ months. Of the patients who died having 
been on ART $>6$ months, $75 \%$ died in hospital. Both groups could be investigated at a tertiary level. Studies such as this involving the clinical record are important as death certificates and routine surveillance data are less accurate. ${ }^{12}$ There is also an inherent tension between clinical assignment and public health coding of cause of death for the purposes of public health planning, programmes and evaluation. Some clinical causes have been labelled 'garbage coding', including categories such as sepsis and pulmonary embolism since each has an underlying cause to precipitate the chain of events leading to death. ${ }^{13}$ In this study, that underlying cause was AIDS.

In conclusion, MAC bacteraemia was an important cause of death not highlighted in other African studies. Access to treatment of malignancy may improve outcomes. Virological failure and nonHIV-related causes were prominent beyond the first year on ART. Initiating ART at higher CD4 counts should reduce early mortality and increase the relative importance of non-HIV-related causes of death over time. ${ }^{14}$

Acknowledgements. I thank Salome Charalambous from the Aurum Institute, Johannesburg, for assistance with data.
2. Lawn SD, Harries AD, Anglaret X, Myer L, Wood R. Early mortality among adults accessing antiretroviral programmes in sub-Saharan Africa. AIDS 2008;22:1897-1908

3. The antiretroviral therapy cohort collaboration. Causes of death in HIV-1 infected patients treated with antiretroviral therapy, 1996-2006: collaborative analysis of 13 HIV cohort studies. Clin Infect is $2010 ; 50 ; 1387-1396$

4. Castelnuovo B, Manabe YC, Kiragga A, Kamya M, Easterbrook P, Kambugu A. Cause-specific mortality and the contribution of immune reconstitution inflammatory syndrome in the first 3 years after antiretroviral therapy initiation in an urban African cohort. Clin Infect Dis 2009;49:965-972.

5. Pettipher CA, Karstaedt AS, Hopley MJ. Prevalence and clinical manifestations of disseminated Mycobacterium avium complex infection in South Africans with acquired immunodeficiency syndrome. Clin Infect Dis 2001;33:2068-2071.

6. Bonard D, Messou E, Seyler C, Vincent V, Gabillard D, Anglaret X. High incidence of atypical mycobacteriosis in African HIV-infected adults with low CD4 cell counts: a 6-year cohort study in Côte d'Ivoire. AIDS 2004;18:1961-1964.

7. Karakousis PC, Moore RD, Chaisson RE. Mycobacterium avium complex in patients with HIV infection in the era of highly active antiretroviral therapy. Lancet Infect Dis 2004;4:557-565.

8. Chao C, Xu L, Abrams D, et al. Survival of non-Hodgkin lymphoma patients with and without HIV 8. Chao C, Xu L, Abrams D, et al. Survival of non-Hodgkin lymphoma patients with
infection in the era of combined antiretroviral therapy. AIDS 2010;24:1765-1770.

9. Bateganya MH, Stanaway J, Brentlinger PE, et al. Predictors of survival after a diagnosis of nonHodgkin lymphoma in a resource-limited setting: a retrospective study on the impact of HIV infection

10. Sliwa K, Wilkinson D, Hansen C, et al. Spectrum of heart disease and risk factors in a black urban population in South Africa (the Heart of Soweto study): a cohort study. Lancet 2008;371:915-922.

11. Brinkhof MW, Spycher BD, Yiannoutsos C, et al. Adjusting mortality for loss to follow-up: analysis of five ART programmes in sub-Saharan Africa. PLoS One 2010;5:e14149.

12. Nojilana B, Groenewald P, Bradshaw D, Reagon G. Quality of cause of death certification at an academic hospital in Cape town, South Africa. S Afr Med J 2009;99: 648-652.

13. Naghavi M, Makela S, Foreman K, et al. Algorithms for enhancing public health utility of national causes-of-death data. Population Health Metrics 2010;8:9.

14. Hoffman CI, Fielding KL, Johnston V, et al. Changing predictors of mortality over time from cART start: implications for care. J Acquir Immune Defic Syndr 2011;58:269-276.

\section{References}

1. Braitstein P, Brinkhof MW, Dabis F, et al. Mortality of HIV-1 infected patients in the first year of antiretroviral therapy: comparison between low-income and high-income countries. Lancet 2006;367: 817-824.

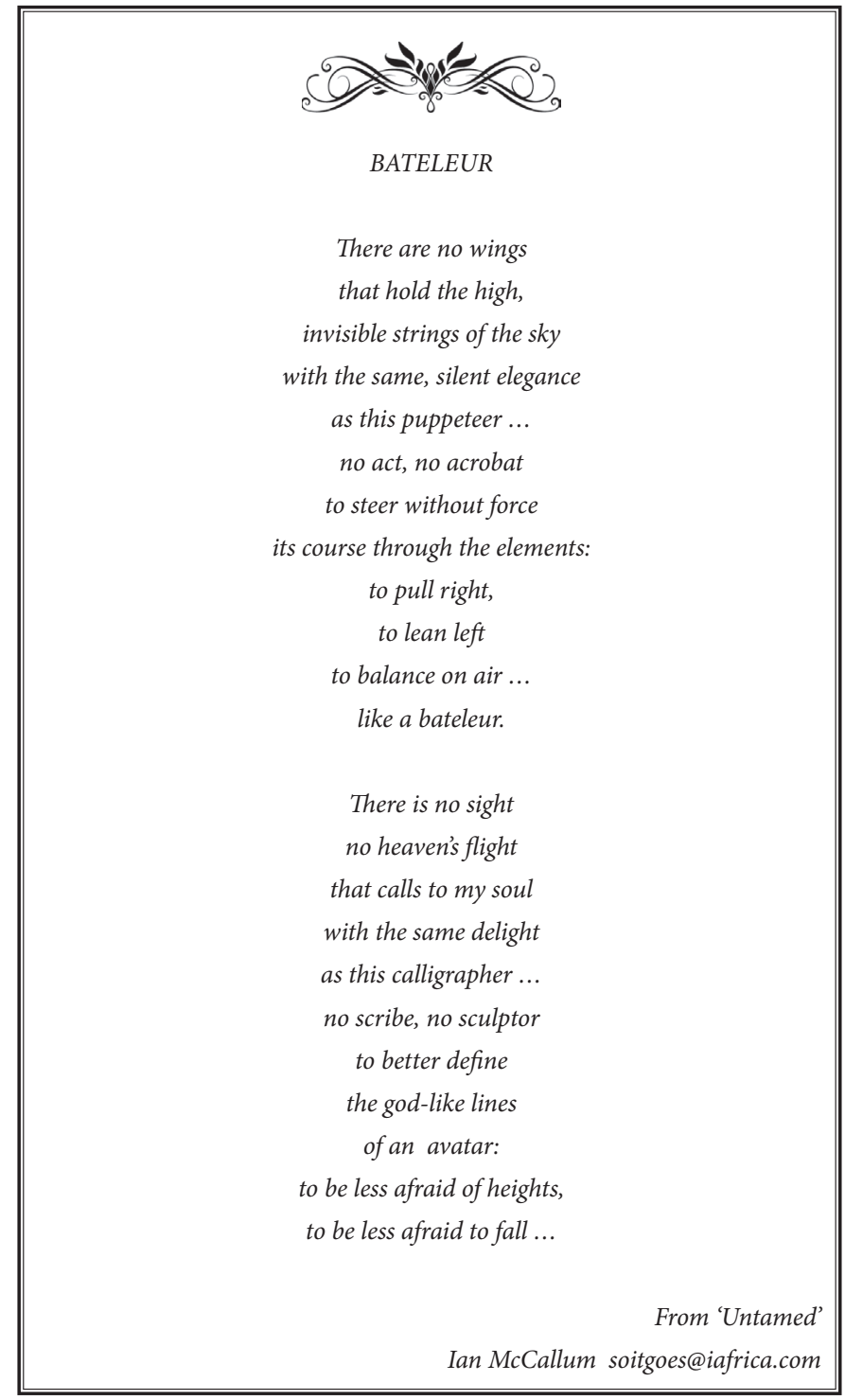

